\title{
Fixed airflow distribution of ventilation network with maximum flow algorithm
}

\author{
Deng Lijun \\ College of Safety Science and Engineering \\ Liaoning Technical University \\ Fuxin , Liaoning, China \\ e-mail: anheihb03dlj@163.com
}

\author{
Liu Jian \\ College of Safety Science and Engineering \\ Liaoning Technical University \\ Fuxin, Liaoning, China \\ e-mail: 1j1961@vip.sina.com
}

\begin{abstract}
Whether there is a feasible flow of ventilation network that meets the demand of fixed airflow and the balance of airflow, when the number, quantities of fixed branches are all uncertain. It is called fixed airflow distribution problem, which was transformed into flow distribution problem. All branches have the upper and lower capacities bounds. The upper and lower capacities of fixed airflow branches were set to their values, while the upper capacities of other branches were set to infinity, and lower capacities were set to zero. Two virtual sources-sink nodes was added to the network, while the fixed branches were splitted into two new branches connected with the virutal source-sink nodes. The classical max flow algorithm was adopted to solve the new network. There was a solution of fixed airflow distribution when the new network had a feasible flow and each splitted branches reached its capacity. At last, the method was analysed by means of two examples.
\end{abstract}

Keywords-ventilation network; fixed airflow; independent circuit; max flow; the upper and lower capacities

\section{INTRODUCTION}

The first step of ventilation net work solution is to distribute airflows that satisfied with the flow conservation law. If there are fixed airflow branches, the traditional methods just consider that fixed airflow branches are all in the remaining tree [1]. But in practical application, fixed airflow branches do not always fall in the remaining tree due to the restrictions of ventilation network topology. A self-adaption method was proposed to estimate the resistance of some tunnels [2]. The key of this method lies in how to determine the existence of the airflow distribution solution, sink the demand of fixed airflow and the balance of airflow, while the parameters of fixed airflow branches such as position, quantity are uncertain.

These problems above are all related to the fixed airflow distribution algorithm. In this paper, a new algorithm was proposed on the basis of max flow algorithm with the upper and lower bounds, compared with the algorithm based on agument path.

\section{RESISTANCE INVERSION BASED ON FIXED AIRFLOW}

Ventilation resistance measurement is an important technical means to obtain ventilation network basic datas such as resistance and airflow. Errors exist in all measuring; therefore some resistances are far from the true values [3]. A self-adaption method was proposed to correct the resistance of some tunnels. Some airflow of tunnels with large erros is fixed artificially, and then we can correct inversely the resistance of these tunnels.

For a single source-sink ventilation network with $n$ branches and $\mathrm{m}$ nodes, we handpick a spanning tree to determine $r=n-m+1$ independent cycles and $r$ linearly independent branches in the remaining tree. So the airflow of any branch can be represented by the linear function of these independent branches [4].

$$
q_{i}=\sum_{s=1}^{r} c_{s i} q_{y s} \quad(i=1, \cdots, n)
$$

In the meanwhile, $\mathrm{r}$ independent cycles are satisfied with the resistance balance law:

$$
f_{s}=\sum_{i=1}^{n} c_{s i} r_{i} q_{i}\left|q_{i}\right|-h_{s}^{\prime}=0, \quad(s=1,2, \cdots, r)
$$

Where $c_{s i}$ is the direction coefficient of ith branch in the sth cycle, $q_{y s}$ is the airflow of the sth independent branche, $\mathrm{m}^{3} / \mathrm{s}, q_{i}$ is the airflow of the $i t h$ branch, $\mathrm{m}^{3} / \mathrm{s}, r_{i}$ is the resistance of the ith branch, $\mathrm{kg} / \mathrm{m}^{7}, h_{s}^{\prime}$ is the additional resistance of the sth independent cycle, $P a$, and $f_{s}$ is the resistance sum of the $s t h$ independent cycle, $P a$.

We can suppose that $\mathrm{k}$ branches with fixed airflow are known. In order to facilitate the description, branches will be renumbered. Fixed airflow branches are $1,2, \cdots, k$ and other branches are $k+1, k+2, \cdots, n$. So the resistance of independent cycles with fixed airflow branches can be detailed as flows:

$$
\begin{aligned}
f_{s} & =\sum_{i=1}^{n} c_{s i} r_{i} q_{i}\left|q_{i}\right|-h_{s}^{\prime} \\
& =\sum_{i=1}^{k} c_{s i} r_{i} q_{i}\left|q_{i}\right|-\left(h_{s}^{\prime}-\sum_{i=k+1}^{n} c_{s i} r_{i} q_{i}\left|q_{i}\right|\right) \\
& =\sum_{i=1}^{k} c_{s i} r_{i} q_{i}\left|q_{i}\right|-g_{s}
\end{aligned}
$$

Where $g_{s}$ is the resistance sum of branches that do not contain fixed airflow branches in the sth independent cycle, $P a$. If the ith branche is in the sth cycle, $c_{s i}= \pm 1$, otherwise $c_{s i}=0$. 
Let $\alpha_{s i}=c_{s i} q_{i}\left|q_{i}\right|$, equation (3) can be simplified as:

$$
f_{s}=\sum_{i=1}^{k} \alpha_{s i} r_{i}-g_{s}
$$

In order to ensure that independent cycles are satisfied with the resistance balance law, let $f_{s}=0$. It is supposed that the number of independent cycles with fixed airflow branches is $l$, so the above euqation can be developed as a linear equations:

$$
\left\{\begin{array}{l}
\alpha_{11} r_{1}+\alpha_{12} r_{2}+\cdots+\alpha_{1 k} r_{k}=g_{1} \\
\alpha_{21} r_{1}+\alpha_{22} r_{2}+\cdots+\alpha_{2 k} r_{k}=g_{2} \\
\cdots \cdots \cdots \\
\alpha_{l 1} r_{1}+\alpha_{l 2} r_{2}+\cdots+\alpha_{l k} r_{k}=g_{l}
\end{array}\right.
$$

The matrix coefficient $A^{l \times k}$ is denoted as follows:

$$
A=\left[\begin{array}{cccc}
\alpha_{11} & \alpha_{12} & \cdots & \alpha_{1 k} \\
\alpha_{21} & \alpha_{22} & \cdots & \alpha_{2 k} \\
\vdots & \vdots & \ddots & \vdots \\
\alpha_{l 1} & \alpha_{l 2} & \cdots & \alpha_{l k}
\end{array}\right]
$$

Let $x=\left(r_{1}, r_{2}, \cdots, r_{k}\right)^{T}, b=\left(g_{1}, g_{2}, \cdots, g_{l}\right)^{T}$, then:

$$
A x=b
$$

According to the theory of linear algebra, the solution of linear equations (7) is denoted as follows [5]:

1) When $r(A)=r(A, b)=k$, equation (7) has a unique solution, the wind resistance of fixed airflow branch is also the only one.

2) When $r(A)=r(A, b)<k$, there are infinitely many solutions of equation (7).

3) When $r(A) \neq r(A, b)$, there is no solution.

\section{FIXED AIRFLOW BALANCE ALGORITHM BASED ON AUGMENTING PATH}

From the perspective of network flow, fixed airflow balance problem can also be treated as flow distribution problem. The capacities of fixed airflow branches are equal to its values while the capacities of other branches are infinite. A fixed airflow balance algorithm based on augmenting path was proposed [2]. It is similar to the maxflow algorithm based on augmenting path. It continually finds augmenting paths and augmenting flow among all paths until there is no such augmenting path in the network.

For the flow network $G=(V, E)$ with a single source-sink node, each branch $(u, v)$ in $\mathrm{E}$ has a capacity c, and $\mathrm{c}$ satisfies the condition $c(u, v) \geq 0$. The capacities of the fixed airflow branches are equal to their fixed its values, while the capacities of other branches are all infinite. Each branch $(u, v)$ is given airflow $f(u, v)$. The key of the augmenting path algorithm is building the residual network $G^{\prime}$, each branch $(u, v)$ in the original network $G$ is mapped as a forward branch $(u, v)$ and a backward branch $(v, u)$. The capacities of the two branches can be denoted as follows [6]:

$$
\left\{\begin{array}{l}
c^{\prime}(u, v)=c(u, v)-f(u, v) \\
c^{\prime}(v, u)=f(u, v)
\end{array}\right.
$$

Let $P$ be an augmenting path, $\delta$ be an augmenting airflow. $\delta$ is denoted as $\delta=\min _{(u, v) \in P}\left\{c^{\prime}(u, v)\right\}$. We can search an augmenting path $\mathrm{P}$ in the residual network $G^{\prime}$, add $\delta$ to the quantities of the forward branches in $\mathrm{P}$, and reduce $\delta$ from the quantities of the backward branches in $\mathrm{P}$. So the cycle repeats until there is no $\mathrm{P}$ can be found in $G^{\prime}$.

Fig .1 shows a ventilation network example taken from literature [2] and TABLE 2 shows the two different augmenting processes and results. The max-flow of the two different augmenting processes is both 120 . Obviously, the search order of augmenting path makes a direct influence on the final results.

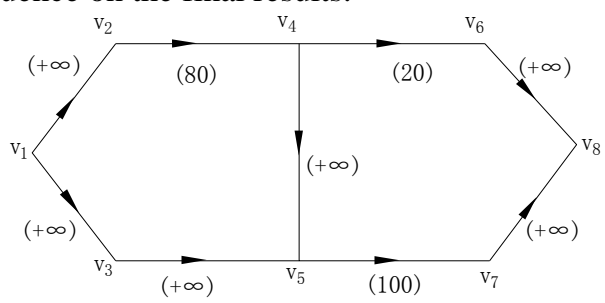

\begin{tabular}{|c|c|}
\hline $\begin{array}{l}\text { Augmenting process proposed in } \\
\text { reference [2] }\end{array}$ & A new augmenting process \\
\hline $\begin{array}{l}\text { 1) augmenting path } \mathrm{P}_{1}=\left\{\mathrm{v}_{1}, \mathrm{v}_{2} \text {, }\right. \\
\left.\mathrm{v}_{4}, \mathrm{v}_{6}, \mathrm{v}_{8}\right\} \text {, augmenting airflow } 20 \text {, } \\
\text { saturated branch }\left(\mathrm{v}_{4}, \mathrm{v}_{6}\right) \\
\text { 2) augmenting path } \mathrm{P}_{2}=\left\{\mathrm{v}_{1}, \mathrm{v}_{2} \text {, }\right. \\
\left.\mathrm{v}_{4}, \mathrm{v}_{5}, \mathrm{v}_{7}, \mathrm{v}_{8}\right\} \text {, augmenting airflow } \\
60 \text {, saturated branch }\left(\mathrm{v}_{2}, \mathrm{v}_{4}\right) \\
\text { 3) augmenting path } \mathrm{P}_{3}=\left\{\mathrm{v}_{1}, \mathrm{v}_{3} \text {, }\right. \\
\left.\mathrm{v}_{5}, \mathrm{v}_{7}, \mathrm{v}_{8}\right\} \text {, augmenting airflow } 40 \text {, } \\
\text { saturated branch }\left(\mathrm{v}_{5}, \mathrm{v}_{7}\right) \\
\text { Conclusions: all fixed airflow } \\
\text { branches are saturated and the } \\
\text { max-flow value is } 120 \text {. }\end{array}$ & $\begin{array}{l}\text { 1) augmenting path } \mathrm{P}_{1}=\left\{\mathrm{v}_{1}, \mathrm{v}_{3}, \mathrm{v}_{5},\right. \\
\left.\mathrm{v}_{7}, \mathrm{v}_{8}\right\} \text {, augmenting airflow } 100 \text {, } \\
\text { saturated branch }\left(\mathrm{v}_{5}, \mathrm{v}_{7}\right) \\
\text { 2) augmenting path } \mathrm{P}_{2}=\left\{\mathrm{v}_{1}, \mathrm{v}_{2}, \mathrm{v}_{4},\right. \\
\left.\mathrm{v}_{6}, \mathrm{v}_{8}\right\} \text {, augmenting airflow } 20 \text {, } \\
\text { saturated branch }\left(\mathrm{v}_{4}, \mathrm{v}_{6}\right) \\
\text { 3) no augmenting paths can be found } \\
\text { Conclusions: fixed airflow branch } \\
\left(\mathrm{v}_{2}, \mathrm{v}_{4}\right) \text { is not saturated, the residual } \\
\text { airflow is 60, and the max-flow } \\
\text { value is } 120 \text {. }\end{array}$ \\
\hline
\end{tabular}

Figure 1. Ventilation network with fixed airflow (from: reference[2], P137)

TABLE I. TWO AUGMENTING PROCESSES OF VENTILATION NETWORK AS SHOWN IN FIG .1

\section{FIXED AIRFLOW BALANCE ALGORITHM BASED ON MAX-FLOW ALGORITHM}

The agumenting path algorithm only took into account the upper bound of the branch capacity. In this paper, both the lower and the upper bound of the branch capacity were taken into account. The upper and lower capacity of the fixed airflow branches is equal to its quantities. So the fixed airflow distribution problem was transformed into determing whether there is a feasible flow of the network limited by the upper and lower capacity bounds. All the flow problems can be solved by the max-flow algorithm.

The classical max-flow algorithm only considers satisfying the upper capacity bound [7]. Besides, the airflow of the branch must satisfy the lower capacity bound in the network that has the upper and lower bounds. In the premise that the flow network has upper and lower bounds, we built an extra network without the source-sink nodes, splitted the lower capacity into a new branch, and 
resort to the max-flow algorithm for determining the feasible flow.

\section{A. Definition of flow network with the upper and lower bounds and the source-sink nodes}

Different from the flow network definition of the traditional max-flow problem, qunatities of the branches in flow network with upper and lower bounds must be greater than or equal to a certain lower bound. For the flow network $G=(V, E)$ with a single source-sink node, s is the Source, and tis the sink node. Each branch $(u, v)$ in $\mathrm{E}$ has a lower capacity bound $b(u, v)$ and an upper capacity bound $c(u, v)$, and they satisfy the equations: $b(u, v) \geq 0$ and $c(u, v) \geq 0$. Each branch $(u, v)$ is denoted by a flow $f(u, v)$. If $\mathrm{f}$ satisfies two conditions as follows, it is a feasible flow of $G$.

(1) Flow balance conditions: $i$ is one of the nodes in the network except the Source $s$ and the sink node $t$, and it must satisfy the equation: $\sum_{(u, i) \in E} f(u, i)=\sum_{(i, v) \in E} f(i, v)$

(2) Capacity bounds conditions: any branch $(u, v)$ in E must satisfy the equation: $b(u, v) \leq f(u, v) \leq c(u, v)$.

\section{B. Feasible flow in the network with the upper and}

lower bounds and without the source-sink nodes

From the perspective of network flow, the flow is a circulation flow, and each node's inflow and outflow are equal, satisfying the flow conservation law[8]. Considering a flow network $C$ with the upper and lower bounds and without the source-sink nodes, it demands that any node should satisfy the airflow balance and the capacity bounds, and find a feasible flow.

The flow of the branch is defined by $f(u, v)$, which must be greater than or equal to the lower capacity.

$$
f(u, v)=b(u, v)+g(u, v)
$$

we can ensure that $f(u, v) \geq b(u, v)$, if $g(u, v) \geq 0$.

$$
g(u, v) \leq c(u, v)-b(u, v)
$$

Let $c^{\prime}(u, v)=c(u, v)-b(u, v)$. We can construct a new flow network $C^{\prime}=(V, E), g(u, v)$ is the flow of the branch. In new network $C^{\prime}$, and $c^{\prime}(u, v)$ is the capacity of the branch.

In order to deal with the lower bounds $b(u, v)$, euqation (11) was put into the airflow balance expression.

$$
\begin{array}{r}
\sum_{(u, i) \in E}[b(u, i)+g(u, i)]=\sum_{(i, v) \in E}[b(i, v)+g(i, v)] \\
\sum_{(i, v) \in E} g(i, v)-\sum_{(u, i) \in E} g(u, i)=\sum_{(u, i) \in E} b(u, i)-\sum_{(i, v) \in E} b(i, v)
\end{array}
$$

The euqation right end is denoted by $m(i)$, which is the sum of the inflows minus the sum of the outflows of the ith node's lower bound.

$$
m(i)=\sum_{(u, i) \in E} b(u, i)-\sum_{(i, v) \in E} b(i, v)
$$

Excluding the excess flow $m(i)$ from the network $C^{\prime}$ in order to make the nodes in the network $C^{\prime}$ satisfy the airflow conservation conditions:

$$
\sum_{(i, v) \in E} g(i, v)=\sum_{(u, i) \in E} g(u, i)
$$

$m(i)>0$ means that the inflow of lower capacity is greater than the flow of lower capacity, and make excess flow $m(i)$ transfer to a new branch $\left(s^{\prime}, i\right)$. The capacity of the new branch is:

$$
c^{\prime}\left(s^{\prime}, i\right)=m(i)
$$

$m(i)<0$ mean that the inflow of lower capacity is less than the flow of lower capacity, and make excess flow transfer to a new branche $\left(i, t^{\prime}\right)$. The capacity of the new branch is:

$$
c^{\prime}\left(i, t^{\prime}\right)=-m(i)
$$

In the network $C^{\prime}$, if any branch's flow was saturated, a feasible flow $g$ must be corresponding with a feasible flow $f$ of the original network $C$.On the contrary, each feasible flow $f$ of the network $C$ must be corresponding with the saturated flow that all branches $g\left(s^{\prime}, i\right)$ and $g\left(i, t^{\prime}\right)$ reached their capacities.

\section{Feasible flow in the network with the upper and lower bounds and with the source-sink node}

By means of the method above, we would like to construct an extra network $G^{\prime}$ as shown in Fig .2, adding a virtual source source $s^{\prime}$ and a virtual sink $t^{\prime}$. Then the branches with the upper and lower bounds were splitted into three new branches according to euqation (17) and (18). The first and second branches are $\left(s^{\prime}, v\right)$ and $\left(u, t^{\prime}\right)$. The third branch is $(u, v)$, the capacity of that is equal to $c^{\prime}(u, v)=c(u, v)-b(u, v)$. At last, the source and the sink are connected by a new branch $(t, s)$, making the network be of no source-sink.

So the task is to find a feasible flow from $s^{\prime}$ to $t^{\prime}$. If all the outflow branches of $s^{\prime}$ are saturated, there is a feasible flow in the original network $G$, which is satisfied with the demand of fixed air flow and the airflow balance.

The classic maximum flow algorithm was adopted to find a feasible flow. There are many max-flow algorithms such as Ford-Fulkerson method, Shortest Augmenting Path (SAP), Edmonds-Karp Algorithm, Dinic Algorithm, Improved SAP Algorithm, Maximum Capacity Path Algorithm, Capacity Scaling Algorithm, Push-Relabel Algorithm and so on. Those maximum flow algorithms are very mature and perfect. Please reference the literature [9, $10]$. 


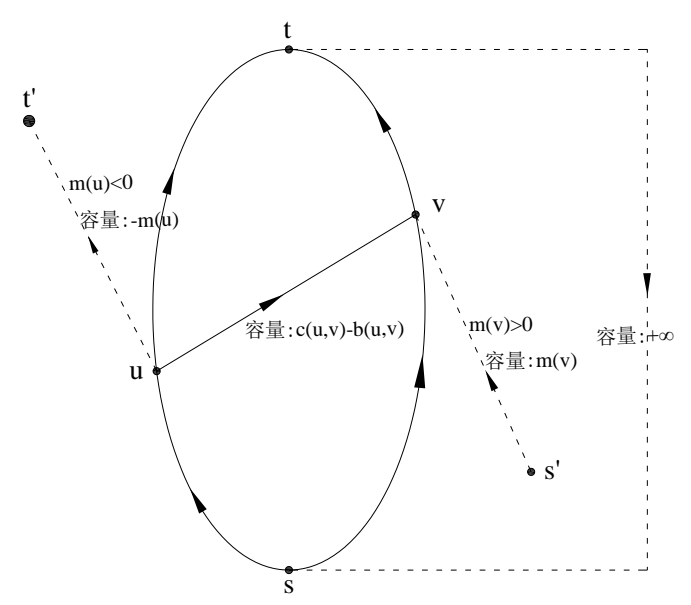

Figure 2. Schematic diagram of additional network $\mathrm{G}^{\prime}$

\section{4 EXAMPLE ANALYSIS}

The example as previously shown in Fig .1 was a single source-sink network, the source and sink respectively was $\mathrm{v}_{1}$ and $\mathrm{v}_{8}$. The upper and lower bounds of the branch capacities were shown in TABLE II. The new branches were added into the extra network according to euqation (17) and (18), shown in TABLE III. The final extra network was shown in Fig .3. The capacities of all the branches in the extra network were shown in TABLE IV.

The maximum flow was used to find a feasible flow from $s^{\prime}$ to $t^{\prime}$, then the result was shown in TABLE V. It could conclude that all the ourflow branches of the virtual source node $s^{\prime}$ were saturated, so there was a feasible solution of air flow, satisfying with both the demand of fixed airflow and the flow balance. The result of fixed air flow distribution was shown in TABLE VI.

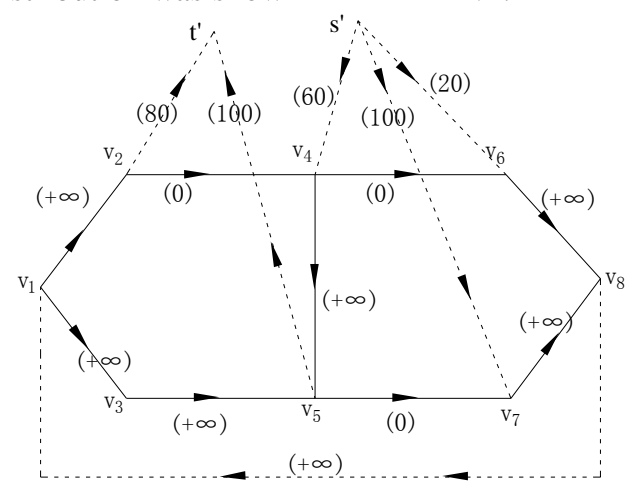

Figure 3. The extra network

TABLE II. THE UPPER AND LOWER BOUNDS OF BRANCH CAPACITY IN VENTILATION NETWORK

\begin{tabular}{llllll}
\hline Source & Target & Bounds & Source & Target & Bounds \\
\hline $\mathrm{v}_{2}$ & $\mathrm{v}_{4}$ & $80-80$ & $\mathrm{v}_{3}$ & $\mathrm{v}_{5}$ & $0-+\infty$ \\
$\mathrm{v}_{4}$ & $\mathrm{v}_{6}$ & $20-20$ & $\mathrm{v}_{4}$ & $\mathrm{v}_{5}$ & $0-+\infty$ \\
$\mathrm{v}_{5}$ & $\mathrm{v}_{7}$ & $100-100$ & $\mathrm{v}_{6}$ & $\mathrm{v}_{8}$ & $0-+\infty$ \\
$\mathrm{v}_{1}$ & $\mathrm{v}_{2}$ & $0-+\infty$ & $\mathrm{v}_{7}$ & $\mathrm{v}_{8}$ & $0-+\infty$ \\
$\mathrm{v}_{1}$ & $\mathrm{v}_{3}$ & $0-+\infty$ & & & \\
\hline
\end{tabular}

TABLE III. THE UPPER AND LOWER BOUNDS OF FIXED BRANCH CAPACITY AFTER BEING SPLITTED

\begin{tabular}{ccccc}
\hline Node & $\begin{array}{c}\text { The sum of } \\
\text { inflows }\end{array}$ & $\begin{array}{c}\text { The sum of } \\
\text { the outflows }\end{array}$ & m(u) & $\begin{array}{c}\text { Add the branch } \\
\text { and capacity }\end{array}$ \\
\hline$v_{2}$ & 0 & 80 & -80 & $\mathrm{v} 2->\mathrm{t}^{\prime}, \mathrm{c}^{\prime}=80$ \\
$\mathrm{v}_{4}$ & 80 & 20 & 60 & $\mathrm{~s}^{\prime}->\mathrm{v} 4, \mathrm{c}^{\prime}=60$ \\
$\mathrm{v}_{5}$ & 0 & 100 & -100 & $\mathrm{v} 5->\mathrm{t}^{\prime}, \mathrm{c}^{\prime}=100$ \\
$\mathrm{v}_{6}$ & 20 & 0 & 20 & $\mathrm{~s}^{\prime}->\mathrm{v} 6, \mathrm{c}^{\prime}=20$ \\
$\mathrm{v}_{7}$ & 100 & 0 & 100 & $\mathrm{~s}^{\prime}->\mathrm{v} 7, \mathrm{c}^{\prime}=100$ \\
\hline
\end{tabular}

TABLE IV. BRANCH CAPACITIES OF THE EXTRA NETWORK

\begin{tabular}{cccccc}
\hline Source & Target & Capacity & Source & Target & Capacity \\
\hline $\mathrm{s}^{\prime}$ & $\mathrm{v}_{4}$ & 60 & $\mathrm{v}_{1}$ & $\mathrm{v}_{2}$ & $+\infty$ \\
$\mathrm{s}^{\prime}$ & $\mathrm{v}_{6}$ & 20 & $\mathrm{v}_{1}$ & $\mathrm{v}_{3}$ & $+\infty$ \\
$\mathrm{s}^{\prime}$ & $\mathrm{v}_{7}$ & 100 & $\mathrm{v}_{3}$ & $\mathrm{v}_{5}$ & $+\infty$ \\
$\mathrm{v}_{2}$ & $\mathrm{t}^{\prime}$ & 80 & $\mathrm{v}_{4}$ & $\mathrm{v}_{5}$ & $+\infty$ \\
$\mathrm{v}_{5}$ & $\mathrm{t}^{\prime}$ & 100 & $\mathrm{v}_{6}$ & $\mathrm{v}_{8}$ & $+\infty$ \\
$\mathrm{v}_{8}$ & $\mathrm{v}_{1}$ & $+\infty$ & $\mathrm{v}_{7}$ & $\mathrm{v}_{8}$ & $+\infty$
\end{tabular}

TABLE V. THE FEASIBLE FLOW OF EXTRA NETWORK BY MEANS OF THE MAXIMUM FLOW ALOGIRTHM

\begin{tabular}{ccccc}
\hline Source & Target & Capacity & Flows & Whether saturated \\
\hline $\mathrm{s}^{\prime}$ & $\mathrm{v}_{4}$ & 60 & 60 & Yes \\
$\mathrm{s}^{\prime}$ & $\mathrm{v}_{6}$ & 20 & 20 & Yes \\
$\mathrm{s}^{\prime}$ & $\mathrm{v}_{7}$ & 100 & 100 & Yes \\
$\mathrm{v}_{2}$ & $\mathrm{t}^{\prime}$ & 80 & 80 & Yes \\
$\mathrm{v}_{5}$ & $\mathrm{t}^{\prime}$ & 100 & 100 & Yes \\
$\mathrm{v}_{8}$ & $\mathrm{v}_{1}$ & $+\infty$ & 120 & No \\
$\mathrm{v}_{1}$ & $\mathrm{v}_{2}$ & $+\infty$ & 80 & No \\
$\mathrm{v}_{1}$ & $\mathrm{v}_{3}$ & $+\infty$ & 40 & No \\
$\mathrm{v}_{3}$ & $\mathrm{v}_{5}$ & $+\infty$ & 40 & No \\
$\mathrm{v}_{4}$ & $\mathrm{v}_{5}$ & $+\infty$ & 60 & No \\
$\mathrm{v}_{6}$ & $\mathrm{v}_{8}$ & $+\infty$ & 20 & No \\
$\mathrm{v}_{7}$ & $\mathrm{v}_{8}$ & $+\infty$ & 100 & No \\
\hline
\end{tabular}

TABLE VI. THE FIXED AIR FLOW DISTRIBUTION

\begin{tabular}{cccccc}
\hline Source & Target & Airflow & Source & Target & Airflow \\
\hline $\mathrm{v}_{2}$ & $\mathrm{v}_{4}$ & 80 & $\mathrm{v}_{3}$ & $\mathrm{v}_{5}$ & 40 \\
$\mathrm{v}_{4}$ & $\mathrm{v}_{6}$ & 20 & $\mathrm{v}_{4}$ & $\mathrm{v}_{5}$ & 60 \\
$\mathrm{v}_{5}$ & $\mathrm{v}_{7}$ & 100 & $\mathrm{v}_{6}$ & $\mathrm{v}_{8}$ & 20 \\
$\mathrm{v}_{1}$ & $\mathrm{v}_{2}$ & 80 & $\mathrm{v}_{7}$ & $\mathrm{v}_{8}$ & 100 \\
$\mathrm{v}_{1}$ & $\mathrm{v}_{3}$ & 40 & - & - & -
\end{tabular}

Let's modify the network shown in Fig .1, the fixed air flow of the branch $\left(\mathrm{v}_{5}, \mathrm{v}_{7}\right)$ is 50 . Obviously, there was not a feasible solution for fixed air flow distribution. The extra network was shown in Fig. 4 and the result of the max-flow algorithm was shown is TABLE VII. We found that the branch (s', v4) was not saturated, so there was not a resonable solution to meet the demand of fixed air flows and the airflow balance. 


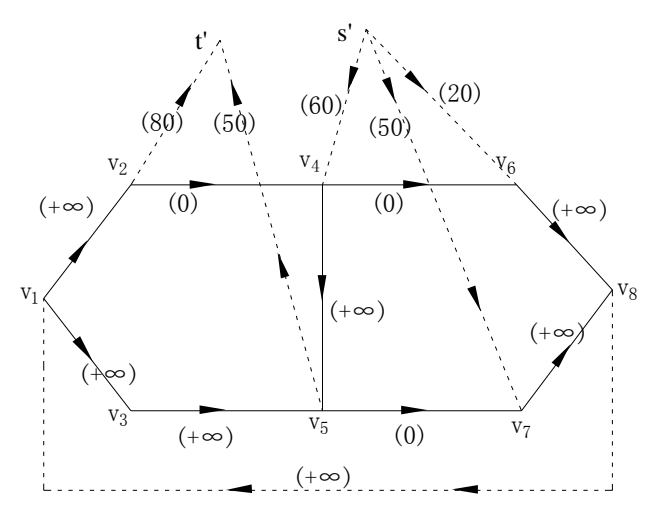

Figure 4. The extra network

TABLE VII. THE FEASIBLE FLOW OF EXTRA NETWORK BY MEANS OF THE MAXIMUM FLOW ALOGIRTHM

\begin{tabular}{ccccc}
\hline Source & Target & Capacity & Flows & Whether saturated \\
\hline $\mathrm{s}^{\prime}$ & $\mathrm{v}_{4}$ & 60 & 50 & No \\
$\mathrm{s}^{\prime}$ & $\mathrm{v}_{6}$ & 20 & 20 & Yes \\
$\mathrm{s}^{\prime}$ & $\mathrm{v}_{7}$ & 50 & 50 & Yes \\
$\mathrm{v}_{2}$ & $\mathrm{t}^{\prime}$ & 80 & 20 & No \\
$\mathrm{v}_{5}$ & $\mathrm{t}^{\prime}$ & 50 & 50 & Yes \\
$\mathrm{v}_{8}$ & $\mathrm{v}_{1}$ & $+\infty$ & 70 & No \\
$\mathrm{v}_{1}$ & $\mathrm{v}_{2}$ & $+\infty$ & 20 & No \\
$\mathrm{v}_{1}$ & $\mathrm{v}_{3}$ & $+\infty$ & 0 & No \\
$\mathrm{v}_{3}$ & $\mathrm{v}_{5}$ & $+\infty$ & 0 & No \\
$\mathrm{v}_{4}$ & $\mathrm{v}_{5}$ & $+\infty$ & 50 & No \\
$\mathrm{v}_{6}$ & $\mathrm{v}_{8}$ & $+\infty$ & 20 & No \\
$\mathrm{v}_{7}$ & $\mathrm{v}_{8}$ & $+\infty$ & 50 & No \\
\hline
\end{tabular}

VI. CONCLUSION

The algorithm of fixed airflow distribution based on augmenting path is similar with the classical maximum flow algorithm. The drawback of this algorithm is that only the upper bound of branch capacity is taken into account. So the search order of augmented paths directly influences the result of fixed air distribution.

The fixed air flow distribution problem was transformed into finding a feasible flow, which can be solved by means of the max-flow alogirhm with the upper and lower bounds. In order to use the classic max-flow algorithm, we made a little change to the flow network, adding two virtual source-sink nodes, splitting the excess flow into new branches. Then the conditions of applying the classic max-flow algorithm were also reached. The classic max-flow algorithm is very mature and quick, so the fixed air flow distribution problem can be well solved.

\section{REFERENCES}

[1] CHEN Kaiyan. The Theroy and Application of Mine Ventilation System Optimization [M]. Jiangsu: China University of Mining and Technology Press, 2003, 06: 71-87.

[2] SU Qingzheng, LIU Jian. The Theroy and Practice of Mine Ventilation Simulation [M]. Beijing: China Coal Industry Publishing House, 2006: 116-180.

[3] WEI Lianjiang. Topology theory of mine ventilation network[J]. Procedia Earth and Planetary Science,2009,11:
[4] CHEN Kaiyan, ZHAO Yihui. The Correlated Adjustment of Condition Equations for Resistance Measurement of Mine Ventilation Network[J]. Journal of China University of Mining \& Teehnology, 1994, 01: 80-89.

[5] LI Shangzhi. Linear Algebra[M]. Beijing: Higher Education Press, 2006, 5: 184-187.

[6] RAVINDRA K, THOMAS L, JAMES B. Network Flows: Theory, Algorithms, and Applications [M]. China Machine Press, 2005: 168-191.

[7] ZHANG Xianchao, CHEN Guoliang, Wan Yinyu. Research on the Maximum Network Flow Problem[J]. Journal of Computer Research and Development, 2003, 09: 1281-1292.

[8] RIDHA A, MAHJOUB B, THOMAS S. Max Flow and Min Cut with bounded-length paths: complexity, algorithms, and approximation[J]. Mathematical Programming, 2010, 07: 12411250.

[9] THOMAS H, CHARLES E, RONALD L. Introduction to Algorithms [M]. China Machine Press, 2009: 396-432.

[10] SVEN O. KRUMKE, CHRISTIANE Zeck. Generalized max flow in series-parallel graphs[J]. Discrete Optimization, 2013, 19: 452459. 